\title{
An Efficient Antimalware System Using Neural Networks with Support Vector Machine and Discriminative Common Vector
}

\begin{tabular}{|c|} 
M. Sornam \\
Dept. of Computer Science \\
University of Madras \\
Chennai, India \\
*-Corresponding author \\
P. Balamurugan ${ }^{2}$ \\
Dept. of Computer Science \\
University of Madras \\
Chennai, India
\end{tabular}

\section{ABSTRACT}

There is a plenty of malicious software available in internet. Internet users are in high risk in downloading such malicious software. The existing antivirus packages lacks in generalization ability. So an efficient antimalware system was proposed using Neural Networks. The antimalware system is treated as a classification problem. There are two approaches considered for the classification viz., classifying the malware using MLP with Support Vector Machine and by using Radial Basis Function neural network with Discriminative Common Vector (DCV). The malware binaries are first converted into grey scale images and then the images are classified using MLP-SVM and RBF-DCV networks. Based on the results it was found that RBF with DCV network results in better score for classifying malware images with $89.16 \%$. The next stage towards building an anti-malware system shall be to explore other better neural network models for classification.

General Terms:Image Classification

Keywords:ANN, Multilayer Perceptron, Support Vector Machine, Radial Basis Function, Discriminative Common Vector

\section{INTRODUCTION}

With the increased use of internet technology, there is lot of software packages available in the internet for free of cost. There is lot of risk involved in downloading those software packages. The chances of getting a malicious software package are very high. The malicious software which got downloaded could be a malware. The malware is software designed intentionally to create damage the computer system or to network. So there is a need to classify software packages which are legitimate or not. Artificial Neural Network (ANN) is applied to solve many problems of classification and prediction. ANN has an ability to learn the parameters and exhibits a generalization for classification. There is lot of Neural Network model available in the literature. Multi-Layer Perceptron (MLP) is one of the feed forward artificial neural network and used in classifying the data which are not linearly separable. Radial Basis Function Neural Network uses Radial Basis Function as an activation function for converting non-linear data points to linear data points. Thus the output produced is a linear combination of RBF and the input parameters. Both these network models are applied to classify the malware into different classes of malware families. 
DOI: https://dx.doi.org/10.26808/rs.ca.i8v5.01 International Journal of Computer Application (2250-1797)

Issue 8 Volume 5, Sep. - Oct. 2018

There are two steps concerned in this comparison study. The first step is to visualize the malware binaries. The malware is software and the raw binary data cannot be used directly for classification. Hence the binary data is visualized as a grey scale image. Then the problem is converted into image classification problem. In second step, the visualized image is then classified used two neural network models.

Feature extraction is a predominant step before training the network models. There may be redundant or irrelevant information available in the feature vector, which is not required to for training. Discriminative Common Vector is a feature extraction method, applied to extract important features from the input feature set.

During this comparison, the following parameters are measured. Accuracy, F1 Score and Recall. A comparison is made based on these measurement parameters to decide which network model is efficient for classifying malware among its variants.

Rest of the paper is organized as follows, Section 2 briefly explains about the related work done for malware visualization and classification, Section 3 contains details about the data set used in the study, Section 4 describes malware visualization technique, Section 5 explains about data processing, Section 6 provides details about the neural network model used in the study, Section explains the method of DCV, Section 7 discusses the results and Section 8 concludes with the summary of the research.

\section{RELATED WORK}

There are various methods available in literature for analyzing the malware. One such method is to extract binary signatures from malware and comparing it with the available database of legitimate footprints. This method is inefficient because of the increase of number of malwares. The other way is perform static and dynamic code analysis. Both of these approaches are time consuming and not efficient in determining the malicious part due to various limitations.

A novel approach was proposed by L. Nataraj et al to characterize and to analyze the malware [1]. The malware binaries are represented as a matrix and visualized as an image. During this visualization, it was observed that there are visual similarities in texture of image for the malwares belonging to same family.

There are several techniques proposed for classifying malware. Behavior analysis based on the feature set was proposed by Rieck et al [2]. The behavior of malwares was monitored in a test environment and the behavior was formulated as feature set. Support Vector Machine was used for classification.

Tian et al, used program length for Trojan classification [3]. Along with the length, the printable string information available in the malware was used for classification [4]. Park et al proposed a method to use behavioral graph for classification [5].

L. Nataraj et al [1] used a method of k-nearest neighbors with 10 cross validations using Euclidian distance over the malware images. The method was evaluated with 1713 malware images of 8 families and achieved an accuracy of $98 \%$

With the generalization capability of deep learning, it was employed to create an intelligent anti-malware system proposed by Aarap et al [6]. The deep learning models used Support Vector Machine (SVM) as classifier.

Another very import step before classification is, features extraction from the input vectors. There are lot approaches proposed for feature extraction. Principal Component Analysis (PCA) is a method in which the features are represented with a linear combination of weighted eigenvector [9]. PCA uses pixel wise correlation and this may be not sufficient to classify Malware images. Because malware images belonging to same family exhibit lot of similarities. 
DOI: https://dx.doi.org/10.26808/rs.ca.i8v5.01 International Journal of Computer Application (2250-1797)

Issue 8 Volume 5, Sep. - Oct. 2018

Linear Discriminant Analysis (LDA) uses Fisher Liner Discriminant Model for features extraction which solves the limitations of Eigen vectors of PCA [10].

A new face recognition method using Discriminative Common Vector (DCV) was proposed by Cevkalp et al [11]. DCV uses within-class scatter matrix of input data and Gram-Schmidt orthogonalization procedure was used to get DCV.

Radial Basis Function network model applied to problems of classification, time-series prediction, function approximation etc. T. Kathirvalavakumar et al [7] proposed a method to use RBF network for face recognition with reduced feature set using DCV.

\section{DATA SET}

The dataset which is used in this comparison study is on Malimg dataset [1]. The dataset consists of 9,339 malware samples from 25 different malware families. Table 1. lists the distribution of malware families and number of samples in the Malimg dataset [1]

Table 1. Malware Families available in Malimg Dataset [1]

\begin{tabular}{|l|l|l|l|}
\hline No. & \multicolumn{1}{|c|}{ Class } & \multicolumn{1}{|c|}{ Family } & $\begin{array}{c}\text { No. of } \\
\text { Samples }\end{array}$ \\
\hline 1 & Worm & Allaple.L & 1591 \\
\hline 2 & Worm & Allaple.A & 2949 \\
\hline 3 & Worm & Yuner.A & 800 \\
\hline 4 & PWS & Lolyda.AA 1 & 213 \\
\hline 5 & PWS & Lolyda.AA 2 & 184 \\
\hline 6 & PWS & Lolyda.AA 3 & 123 \\
\hline 7 & Trojan & C2Lp.P & 146 \\
\hline 8 & Trojan & C2Lp.gen!g & 200 \\
\hline 9 & Dialer & Instantaccess & 431 \\
\hline 10 & Trojan & Swizzot.gen!I & 132 \\
\hline 11 & $\begin{array}{l}\text { Trojan } \\
\text { Downloader }\end{array}$ & Swizzot.gen!E & 128 \\
\hline 12 & Worm & VB.AT & 408 \\
\hline 13 & Rogue & Fakerean & 381 \\
\hline 14 & Trojan & Alueron.gen!J & 198 \\
\hline 15 & Trojan & Malex.gen!J & 136 \\
\hline 16 & PWS & Lolyda.AT & 159 \\
\hline 17 & Dialer & Adialer.C & 125 \\
\hline 18 & Trojan & Wintrim.BX & 97 \\
\hline 19 & Downloader & & \\
\hline 20 & $\begin{array}{l}\text { Trojan } \\
\text { Downloader }\end{array}$ & Dialplatform.B & 177 \\
\hline 21 & $\begin{array}{l}\text { Trojan } \\
\text { Downloader }\end{array}$ & Obfuscator.AD & 142 \\
\hline 22 & Backdoorx & Agent.FYI & 116 \\
\hline 23 & Worm:AutoIT & Autorun.K & 106 \\
\hline 24 & Backdoor & Rbot!gen & 158 \\
\hline 25 & Trojan & Skintrim.N & 80 \\
\hline & & & 162 \\
\hline
\end{tabular}




\section{DATA VISUALIZATION}

The malware software is visualized as an image. The provided malware binary is read as a vector of 8 bit unsigned integers and then arranged into a 2D array. This arrangement can be visualized as a gray scale image in the range of 0 to 255 (255: white and 0: black). The width of the image is fixed and the height is varied based on the malware size.

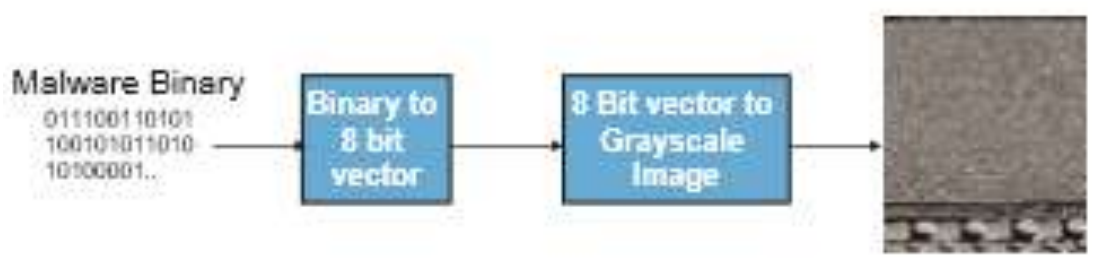

Fig 1: Visualizing malware as a grayscale image [1]

Fig. 2 shows an example image of a common Trojan downloader, Dontovo A, which downloads and executes arbitrary files [8]. From the image, the different sections or fragments are clearly seen.

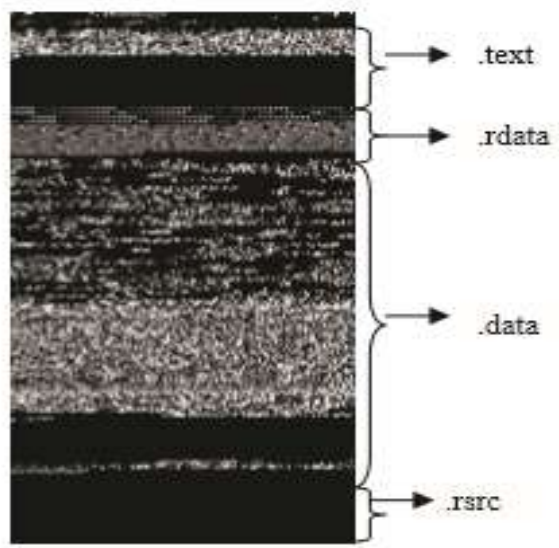

Fig. 1 Various sections of Trojan: Dontovo.A [1]

\section{DATA PROCESSING}

The malware images were resized to a 2-dimensional matrix of $32 \times 32$, and were compressed into 1 x 1024-size array. Each feature array was then labelled with its corresponding indexed malware family name $(0-24)$. Then the features were standardized using the following equation:

$$
z=\frac{X-\mu}{\sigma}
$$

Where $\mathrm{X}$ is the feature to be standardized, $\mu$ is its mean value, and $\sigma$ is its standard deviation.

\section{NEURAL NETWORK MODEL}

This section presents the Support Vector Machine (SVM) and Radial Basis Function network models used in this comparison study.

\subsection{Support Vector Machine (SVM)}

SVM learns the parameters $\mathrm{w}$ and $\mathrm{b}$ by solving the following constrained optimization problem:

$$
\min \frac{1}{p} \mathbf{w}^{T} \mathbf{w}+C \sum_{i=1}^{p} \xi_{i}
$$


Subjected to,

$$
\begin{array}{r}
\text { s.t } y_{i}^{\prime}(\mathbf{w} \cdot \mathbf{x}+b) \geq 1-\breve{\xi}_{i} \\
\xi_{i} \geq 0, i=1, \ldots, p
\end{array}
$$

Where $\mathrm{w}^{\mathrm{T}} \mathrm{w}$ is the $\mathrm{L}_{1}$ norm, $\mathrm{C}$ is the penalty parameter and $\xi$ is a cost function. The corresponding unconstrained optimization problem of Eq. 2 is given by Eq.5.

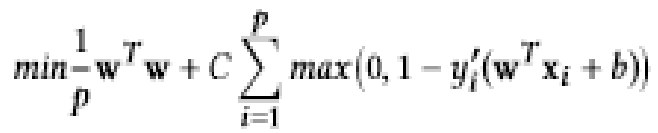

Where $\mathrm{y}^{\prime}$ is the actual label, and $w^{T} x_{i}+b$ is the predictor function. This equation is known as $\mathrm{L}_{1}-\mathrm{SVM}$, with the standard hinge loss. Its derivative is $\mathrm{L}_{2}-\mathrm{SVM}$ given by:

$$
\min \frac{1}{p}\|\mathbf{w}\|_{2}^{2}+C \sum_{i=1}^{p} \max \left(0,1-y_{i}^{\prime}\left(\mathbf{w}^{T} \mathbf{x}_{i}+b\right)\right)^{2}
$$

Where $\|w\|_{2}$ is the Euclidean norm (also known as $\mathrm{L}_{2}$ norm), with the squared hinge loss.

\subsection{Multilayer Perceptron with SVM}

MLP consists of hidden layers. LeakyRelu is the activation function used. The learning parameters were learnt by L2-SVM. The computed loss is minimized using Adam Optimization. argmax function is used for label prediction.

\subsection{Radial Basis Function}

Radial Basis Function (RBF) Neural Network is used for classifying the malware images. RBF contains three layers: input, hidden and output layers. The number of nodes in the input layer corresponds to feature set. There are 1024 features in the data set. The input neurons are normalized and passed as input to the hidden layer. The hidden layer neurons used Gaussian function as basis function and the computed basis function output are passed to the output layer for classification.

The hidden layer output (basis function) is computed as

$$
\boldsymbol{\varphi}_{j}(\boldsymbol{X})=\exp \left\{-\frac{\|\boldsymbol{X}-\boldsymbol{\mu}\|^{2}}{\boldsymbol{\sigma}^{2}}\right\}
$$

Where $X=(x 1, x 2, \ldots x n)^{T}$ is the normalized input vector, $\mu$ is the center and $\sigma$ is the width. The output of the output layer is computed as,

$$
y_{i}=\sum_{j=1}^{k} w_{j i} \varphi_{j}(X)
$$

Where $\mathrm{k}$ is the number of hidden neurons, wji are the weights connecting the hidden layer neuron $\mathrm{j}$ and output layer neuron $\mathrm{i}$. The weights are adjusted using the formula,

$$
w(\mathrm{t}+1)=w(\mathrm{t})+\lambda\left(d_{i}-y_{i}\right) \varphi_{j}(X)
$$

Where $\lambda$ is a positive learning rate parameter and $d_{i}$ is the desired output.

\subsubsection{Algorithm for RBF Network}

The training algorithm for RBF is as follows:

Step 1. Initialize Weights by generating random numbers

Step 2. $\quad$ Feed the network with Input Feature Set and Expected output values

Step 3. Compute hidden layer output value using the Eq. 7 for each of the input data set. 
DOI: https://dx.doi.org/10.26808/rs.ca.i8v5.01 International Journal of Computer Application (2250-1797)

Issue 8 Volume 5, Sep. - Oct. 2018

Step 4. Using Eq. 8, compute output layer output value

Step 5. Compute the Error by finding difference between expected and actual output

values

Step 6. Using Eq. 9, update hidden layer weights.

Step 7. Compute the output of the output layer

Step 8. $\quad$ Calculate sum of squared error of the RBF network

Step 9. Repeat steps $3-8$ for all of the malware images

Step 10. Repeat steps $3-9$ until the error value converge to minimum acceptance value.

\subsection{DISCRIMINATIVE COMMON VECTOR}

Discriminative Common Vector is a feature extraction mechanism for extracting the most discriminative features from the dataset. This feature extraction is necessary to avoid using redundant or irrelevant data and to recover the salient features which have more discriminative power. This is very useful for malware image dataset because the images are visually looks as much as similar and need very discriminative features to classify images. Within-class scatter matrix method is applied to construct discriminative feature vector. With the extracted features of each class, a common vector is attained in the direction of eigenvectors corresponding to the non-zero Eigen values of within-class scatter matrix. The obtained new feature vector is known as Discriminative Common Vector (DCV) and shall be used for classification.

Consider, there are $\mathrm{C}$ classes available in the training dataset with each class containing $\mathrm{N}$ samples. Let $x_{m}^{i}$ denotes $m^{\text {th }}$ sample of $i^{\text {th }}$ class. The within-class scatter matrix of the sample dataset constructed to get features vectors is given by [4]

$$
S_{W}=B B^{T}
$$

Where the matrix $\mathrm{B}$ is given by,

$$
B=\left[x_{1}^{1}-\mu_{1}, \ldots, x_{N}^{1}-\mu_{1}, x_{1}^{2}-\mu_{2}, \ldots, x_{N}^{C}-\mu_{C}\right]
$$

Where $x_{i}^{i}$ is $\mathrm{i}^{\text {th }}$ sample of class $\mathrm{j}$ and $\mu_{\mathrm{j}}$ is the mean of the samples of in the $\mathrm{j}^{\text {th }}$ class.

Let $\mathrm{Q}$ be the set of orthonormal eigenvectors corresponds to non-zero Eigen values of $S_{W}$. $Q=\left[\alpha_{1} \ldots, \alpha_{r}\right]$, where $\mathrm{r}$ is the dimension of $S_{W}$.

The common vector is obtained by using:

$$
x_{\text {com }}^{i}=x_{m}^{i}-Q \bar{Q} x_{m}^{i}
$$

Where $m=1 \ldots N$ samples and $i=1 \ldots C$ classes. The discriminative component are calculated for the corresponding non-zero Eigen values using:

$$
\begin{gathered}
J\left(W_{\text {opt }}\right)=\underset{W}{\arg \max }\left[W^{T} S_{c o m} W\right] \\
S_{c o m}=B_{c o m} B_{c o m}^{T} \\
B_{c o m}=\left[x_{c o m}^{1}-\mu_{c o m} \ldots x_{c o m}^{C}-\mu_{c o m}\right]
\end{gathered}
$$

Feature Vector for the training set is calculated using:

$$
\Omega_{i}=W^{T} x_{m}^{i}
$$

The above mentioned steps are summarized as below:

1. Using Eq. 11 compute the value of $B$. Then compute the non-zero Eigen values and corresponding Eigen vectors by using the matrix $B B^{T}$

2. To obtain common vectors, select an input sample from each class and project it into the null space of $S_{w}$. Then compute the value of $x_{\text {com }}^{i}$ using the Eq. 12 
DOI: https://dx.doi.org/10.26808/rs.ca.i8v5.01 International Journal of Computer Application (2250-1797)

Issue 8 Volume 5, Sep. - Oct. 2018

3. Using Eq. 13 and Eq. 14 compute the Eigen vectors $w_{k}$ of $S_{c o m}$, corresponding to the non-zero Eigen values

4. The feature vector of the training set is obtained using Eq. 16

\section{RESULTS AND DISCUSSION}

These experiments were conducted in two stages: (1) Training stage and (2) Testing stage. The Malimg dataset was used in these two stages. $70 \%$ of data were used for training and $30 \%$ used for testing.

The experiments in this proposed work were conducted on a system with Intel (R) Core (TM) i7-6600U CPU @ 2.60GHz, 16 GB RAM with 64 bit operating system, x64 based processor. Table II summarizes the experiment results for various variables. The variables are calculated using the following equations:

$$
F=2 \cdot \frac{P P V \cdot T P R}{P P V+T P R}
$$

$$
\begin{aligned}
& P P V=\frac{\text { True Positive }}{\text { TruePositive }+ \text { False Positive }} \\
& T P R=\frac{\text { True Positive }}{\text { True Positive }+ \text { False Negative }}
\end{aligned}
$$

Eq. 10 gives F1 Score which is the harmonic mean of Precision (Eq. 18) and Recall (Eq. 19) Before the start of the experiment, standardization procedure was applied over the feature dataset. The dataset is standardized using the (Eq. 1). For normalization, the mean and the standard deviation of the feature set are used. The features used are not technically images, they are generated out from malware binary files. For general image dataset, this standardized procedure may not be required. The standardization procedure tries to normalize the feature set with respect to mean and standard deviation of binary values of the malware images.

The normalized dataset is used in both training and test phases. The Malimg dataset consists of 9,339 malware samples from 25 different malware families. The dataset are classified using two different neural network models viz. Multi-Layer Perceptron with Support Vector Machine (MLP-SVM) and Radial Basis Function Network with Discriminative Common Vector (RBF-DCV).

Although SVM was meant for binary classification, it may also be used for multi-nominal classification. There are different ways in which this can be achieved. MLP-SVM uses oneversus-all (OvA) approach. OvA consider a given class as the positive class and other classes as negative class. Using OvA approach along with L2-SVM is used for malware classification

RBF network can employed to solve image classification effectively. DCV is a feature reduction mechanism where in which it tries to build a common vector with relevant and valid data. The obtained common vector is called as DCV. DCV is passed as an input to RBF network models.

The training time for both neural models was also compared. MLP-SVM was just using the normalized dataset whereas RBF-DCV using the reduced feature set. Because of usage of reduced feature set, RBF-DCV model was training in a short duration of time when compared to MLP-SVM. Table 2 summaries the training time of both the neural network models.

The results of both the network models were compared. The comparison shows that RBFDCV outperforms with better classification accuracy. Table 3 summarizes the experiment result of both the neural network models. 
Table 2. Training Time

\begin{tabular}{|c|l|c|}
\hline Variables & MLP-SVM & RBF-DCV \\
\hline Training Time & 12 minutes & 10.2 minutes \\
\hline
\end{tabular}

Table 3. Summary of Experiment Results

\begin{tabular}{|l|l|l|}
\hline \multicolumn{1}{|c|}{ Variables } & $\begin{array}{c}\text { MLP-SVM } \\
{[6]}\end{array}$ & \multicolumn{1}{|c|}{$\begin{array}{c}\text { RBF- } \\
\text { DCV }\end{array}$} \\
\hline Training Accuracy & $99.57 \%$ & $98.56 \%$ \\
\hline Testing Accuracy & $80.46 \%$ & $89.16 \%$ \\
\hline F1 & 0.81 & 0.81 \\
\hline Recall & 0.80 & 0.84 \\
\hline
\end{tabular}

Fig. 3 shows the testing performance of MLP-SVM model. This model had a recall of 0.80 and a F1 score of 0.81 .

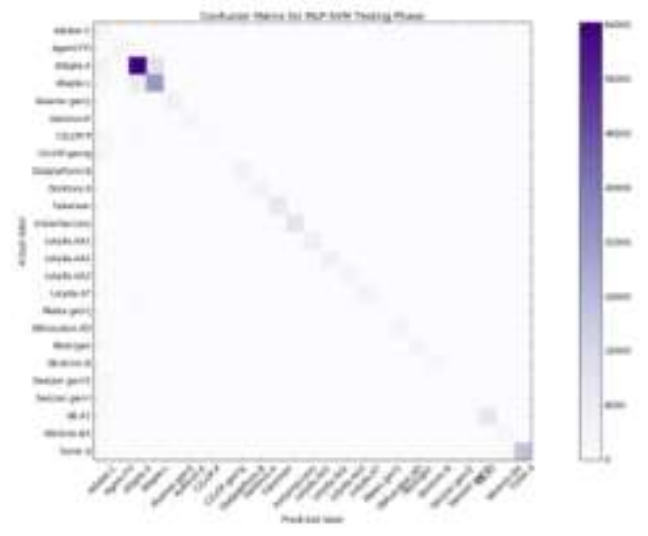

Fig. 2 Confusion Matrix for MLP-SVM

Fig. 4 shows the testing performance of RBF model. This model had a recall of 0.84 and a F1 score of 0.81

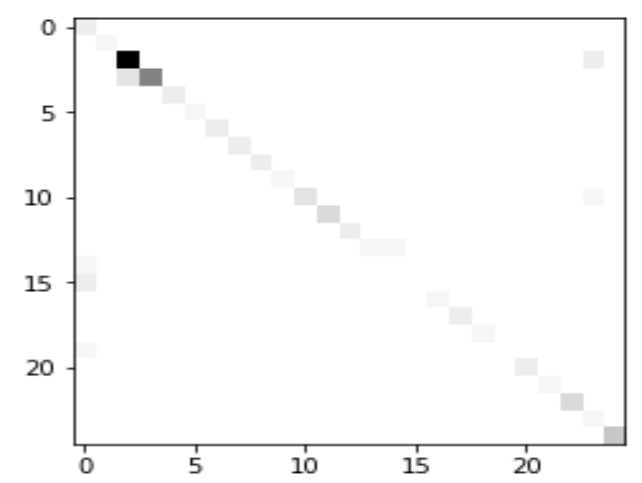

Fig. 3 Confusion Matrix for RBF-DCV

As shown in the confusion matrix, RBF-DCV model performs better classification than MLP-SVM.

\section{CONCLUSION}

The Malimg dataset prepared by [1] consists of malware images which are used for comparison study of malware classification. MLP-SVM and RBF models are employed for classification. As per the results, RBF-SVM had a better scores in malware classification with a test accuracy of $89.16 \%$ 
DOI: https://dx.doi.org/10.26808/rs.ca.i8v5.01 International Journal of Computer Application (2250-1797)

Issue 8 Volume 5, Sep. - Oct. 2018

Furthermore the accuracy can be improved by using any wavelet decomposition methods. Also the running time can be reduced using any other dimensionality reduction techniques. This shall avoid learning unwanted features available in the dataset thereby learning the prime features.

The improvement in the network architecture design by adding more hidden layers, improving other parameter values may provide better perception for improving malware classification. Also such network model can servers as a benchmark to denote the better architecture for the anti-malware system.

\section{REFERENCES}

[1] Lakshmanan Nataraj, S Karthikeyan, Gregoire Jacob and BS Manjunath, Malware Image: Visualization and Automatic Classification, Proceedings of the $8^{\text {th }}$ international symposium on visualization of cyber security, ACM, 4, 2011

[2] Rieck, K. Holz, T. Willems, C. Dussel, P. and Laskov, P, Learning and classification of malware behavior, Fifth Conference on Detection of Intrusions and Malware \& Vulnerability Assessment (DIMVA'08), pp. 108-125, 2008

[3] Tian, R. Batten, L.M, Verstegg. S.C, Function length as tool for malware classification, $3^{\text {rd }}$ International Conference on Malicious and Unwanted Software (MALWARE), 2008

[4] Tian, R. Batten, L. Islam, R. and Versteeg, S, An automated classification system based on the strings on Trojan and virus families, $4^{\text {th }}$ International Conference on Malicious and Unwanted Software: MALWARE, 2009, pp.23-30

[5] Park, Y. Reeves, D. Mulukutla, V. Sundravel, B, Fast malware classification by automated behavioral graph matching, Proc. Of Sixth Annual Workshop on Cyber Security and Information Intelligent Research (CSIIRW'10), 2010

[6] Abien Fred M. Agarap, Francis John Hill Pepito, Towards Building an Intelligent AntiMalware System: A Deep Learning Approach using Support Vector Machine (SVM) for Malware Classification, 2017

[7] T. Kathirvalavakumar, J. Jebakumari Beulah Vasanthi, Features Reduction using Wavelet and Discriminative Common Vector and Recognizing Faces using RBF, International Journal of Computer Applications, vol. 74, No.5, 2013

[8] Microsoft Malware Enclyopedia, http://www.microsoft.com/security/portal/Threat/Encyclopedia/Br owse.aspx

[9] Turk, M. and Pentland, A., Eigenfaces for recognition, J. Cogn. Neuroscience, Vol. 3, pp.71-86, 1991

[10] Swets, D.L. and Wend. J., Using Discriminant Eigen features for Image Retrieval. IEEE Trans. Pattern Analysis and Machine Intelligence, Vol. 18, No. 8, pp. 831-836, 1996

[11] Cevikalp, H., Barkana, B. and Barkana, A, A comparison of the common vector and the discriminative common vector methods for face recognition, Proc. $9^{\text {th }}$ World Multi-Conf. Systemics, Cybern. And Inf., Orlando, FL

[12] M. Sornam, P. Balamurugan, Malware as an Image Classification - ANN approach with Discriminative Common Vector, Intl. Conf on Research Trends in Computing Technologies (ICRTCT-18), 2018, pp.384-389. 